\title{
Magnetoelectric coupling across the interface of multiferroic nanocomposites
}

\author{
Xiefei $\mathrm{Yao}^{1 \dagger}$, Jing $\mathrm{Ma}^{2 \dagger}$, Yuanhua $\mathrm{Lin}^{2 *}$, Ce-wen $\mathrm{Nan}^{2}$ and Jinxing Zhang ${ }^{1 *}$
}

In recent decades, magnetoelectric effect in multiferroic materials has attracted extensive attention owing to the upcoming demands for new-generation multi-functional magnetoelectronic devices, such as transducer, sensor and so on. This gives people a strong push to explore the multiferroic materials with a reduced dimension and effective coupling between electric and magnetic orderings, especially at room temperature. Due to the weak magnetoelectric coupling strength in sing-phase multiferroic materials, scientists start to design nanocomposites and artificial nanostructures with strong coupling among order parameters (lattice, charge, spin and orbital). In this review, we will introduce recent major progresses of magnetoelectric coupling in multiferroic nanocomposites across their interfaces from the following four aspects: strain effect, charge transfer, magnetic exchange interaction and orbital hybridization, based on their coupling mechanisms. Through a full understanding of the above coupling among these orderings, it is possible to achieve the nanoscale modulation of magnetization (ferroelectric polarization) by external electric (magnetic) field. Apart from the magnetoelectric coupling, those artificially functional nanocomposites provide us a platform to explore and study the emerging physical phenomena so that we can design self-assembled nanostructures to tailor novel functionalities in future applications.

\section{BACKGROUND}

Multiferroic materials show the coexistence and coupling of ferroelectric and magnetic orderings. A lot of novel quantum phenomena such as magnetoelectric (ME) effect will emerge with the intrinsic interaction of lattice, charge, spin, and orbital degrees of freedom in such kind of systems [1-5]. For instance, the ferroelectric polarization of the ME materials can be modulated by an external magnetic field; an electric field can also be used to switch the magnetic state. The inter-conversion of electric and magnetic orderings in multiferroic materials can play significant roles in future functional devices. In terms of high-density data storage technology, if the bit state could be written by using an electric field, the energy consump- tion resulted from the current-induced joule heat will be reduced efficiently. In addition, the control of ferroelectric polarization by applying a magnetic field provides us broad opportunities to design functional devices such as sensor, actuator, etc. On account of the demands for the above potential applications in the microelectronic devices, the strong coupling between electric and magnetic orderings becomes one of the central goals pursued by scientists and engineers for decades.

The coupling of magnetism and electrical behaviors was proposed for the first time by Curie [6]. Subsequently, Debye put forward the word "magnetoelectric" to describe the physical phenomenon in 1926 [7]. In the book "Electrodynamics of Continuous Media", Landau and Lifshitz predicted theoretically the linear ME effect due to the coupling between the ferroelectric polarization and magnetic moment in 1960's [8], which had laid a solid physical foundation for the forthcoming studies from the theoretical and experimental points of view. Afterwards, Dzyaloshinskii [9] and Astrov [10] experimentally observed the ME coupling in $\mathrm{Cr}_{2} \mathrm{O}_{3}$ and explained this emerging phenomenon theoretically. Nowadays, more than 80 kinds of single-phase multiferroic ME materials were discovered or synthesized, including systems such as perovskite oxides, borates, manganites, fluorides and so on.

Multiferroic ME materials can be categorized into single-phase multiferroic materials and composite materials. Single-phase multiferroic materials, such as $\mathrm{BiFeO}_{3}$ (BFO), $\mathrm{BiMnO}_{3}(\mathrm{BMO}), \mathrm{TbMnO}_{3}, \mathrm{YMnO}_{3}$ (YMO), HoMn $\mathrm{O}_{3}$ [11-14], etc. exhibit the coexistence of magnetic and ferroelectric orderings. However, the ME coupling in single-phase ME compounds is usually either extremely weak or only exists at extreme conditions (low temperature and high magnetic field). For example, the ME coupling coefficient of $\mathrm{Cr}_{2} \mathrm{O}_{3}$ is $\sim 4.1 \times 10^{-12} \mathrm{~s} \mathrm{~m}^{-1}$ [15]. The ME coefficient of BFO nanowire is about $2.2 \times 10^{-10} \mathrm{~s} \mathrm{~m}^{-1}$ [16]. And for BMO, the application of an ultrahigh magnetic field as large as

Department of Physics, Beijing Normal University, Beijing 100875, China

${ }^{2}$ School of Material Science and Engineering, Tsinghua University, Beijing 100084, China

These authors contributed equally to this work.

* Corresponding authors (emails: linyh@tsinghua.edu.cn (Lin Y); jxzhang@bnu.edu.cn (Zhang J)) 
$9 \mathrm{~T}$ can give a small change of its dielectric constant by only $0.6 \%$ [17]. Up to now, the single-phase ME materials with a strong room-temperature coupling of ferromagnetism and ferroeletricity have not been discovered yet. Alternatively, people start to search for composites with heterogeneous structures consisting of two or more ferroic phases at the nanoscale.

Therefore, in order to achieve a significant magnitude of ME coupling, the key point is to design an appropriate nanostructures consisting of multiple ferroic phases. Owing to the rapid development of advanced thin film growth technologies, high-quality nanostructures can be prepared, e.g., 0-3 mosaic structures, 2-2 horizontal heterostructures (superlattice and multilayers), 1-3 self-assembled structures and so on. Construction of such high-quality heterostructures can significantly enhance the coupling strength across their interfaces. What's more, these nanostructures allow the control of ME effect at nanoscale.

At present, synthesis of the ME nanocomposites with an improved ME coupling which occurs above room temperature are one of the current central focuses. In this review, we will introduce the ME effect according to four coupling mechanisms across the two-phase interface in various composite nanostructures: strain effect, charge transfer, magnetic exchange interaction and orbital hybridization. We hope that this review can help people from various fields to build up a physical picture about the ME coupling from the aspects of controlling lattice, charge, spin and orbital.

\section{COUPLING MECHANISMS OF MAGNETOELECTRIC EFFECT}

\section{Strain effects}

In order to achieve a strong ME coupling, strain-mediation is one of the most conventional and effective ways in multiferroic ME composites and nanostructures. When piezoelectric or ferroelectric materials are assembled with magnetostrictive materials, the magnetic moment (electric polarization) of the material will change through the application of an external electric field (magnetic field). This is a kind of product property by magnetostriction and piezoelectricity, which can be regarded as the transfer of the mechanical energy. The magnitude of ME coupling is defined as below [18]:

$$
\begin{aligned}
& \text { Direct ME effect }=\text { Magnetic/Mechanical } \\
& \times \text { Mechanical/Electric, } \\
& \text { Converse ME effect }=\text { Electric/Mechanical } \\
& \times \text { Mechanical/Magnetic. }
\end{aligned}
$$

In 2004, Zheng et al. [19] reported that pulsed laser deposition (PLD) technique could be an effective tool to fabri- cate the $\mathrm{BaTiO}_{3}$ (BTO)/CoFe $\mathrm{O}_{4}$ (CFO) 1-3 nanocomposite thin films on (001)-oriented $\mathrm{SrTiO}_{3}$ (STO) substrates. BTO is a typical perovskite ferroelectric material [20], while CFO possesses good magnetostrictive properties among oxides [21]. On the (001)-oriented STO substrates, the spontaneous phase separation occurred and resulted in nanoscale columnar heteroepitaxy, as shown in Figs 1a and b. Such a type of composite nanostructures demonstrated not only good ferroelectric (Fig. 1c), piezoelectric (Fig. 1d) and ferromagnetic (Fig. 1e) properties, but also a distinct ME coupling in the vicinity of the ferroelectric Curie temperature: there was an obvious change in magnetization induced by ferroelectric structural transformation, as shown in Fig. 1f. This phenomenon shows that the elastic deformation of BTO during the ferroelectric phase transition has transferred to CFO, which couples with the piezomagnetism of CFO. Although such a behavior did not describe the ME response in this composite nanostructure quantitatively, it, for the first time, provided a direct evidence of the ME coupling in nanoscale composite thin films without substrate clamping. Zavaliche et al. [22] found that the BFO with outstanding ferroelectric property can be an alternative candidate of BTO. In such BFO/CFO 1-3 nanostructures, an external electric field could help to reverse the magnetization using a scanning probe microscope (SPM), demonstrating the nanoscale control of the local magnetization through the electricity-elasticity-magnetism coupling. This also demonstrated the ME coupling by the nanoscale "product" effect and the converse ME coefficient was estimated to be $1.0 \times 10^{-2} \mathrm{G} \mathrm{cm} \mathrm{V}^{-1}$. In those $1-3$ selfassembled spinel-perovskite systems, the nanostructures can be controlled by different substrate orientations [23]. For the BFO and CFO 1-3 nanocomposite, (001)-oriented STO substrates support the rectangular-shaped CFO nanopillars in BFO matrix, while (111)-oriented substrates can stabilize the triangular-shaped $\mathrm{BFO}$ nanopillars in $\mathrm{CFO}$ matrix. The magnetization in BFO/CFO on (001)-oriented STO substrates can be controlled using an external electric field. There was, however, no obvious evidence to show the magnetic-field-driven polarization in BFO/CFO nanocomposites when applying an external magnetic field. This was attributed to the elastic energy required to switch the BFO polarization was higher than magnetoelastic energy by switching the CFO matrix. Very recently, Zhang et al. [24] observed a change of $11.5 \%$ in electric polarization through the effect of external magnetic field in $\mathrm{Pb}(\mathrm{Zr}, \mathrm{Ti})$ $\mathrm{O}_{3}$ (PZT) and CFO nanocomposites with 1-3 free-standing heteropillars.

The above 1-3 nanostructures can help to reduce the substrate constraint during the elastic coupling between ferroelectric and magnetic phases. However, it seems to exist only in limited material systems. The large leak- 
a
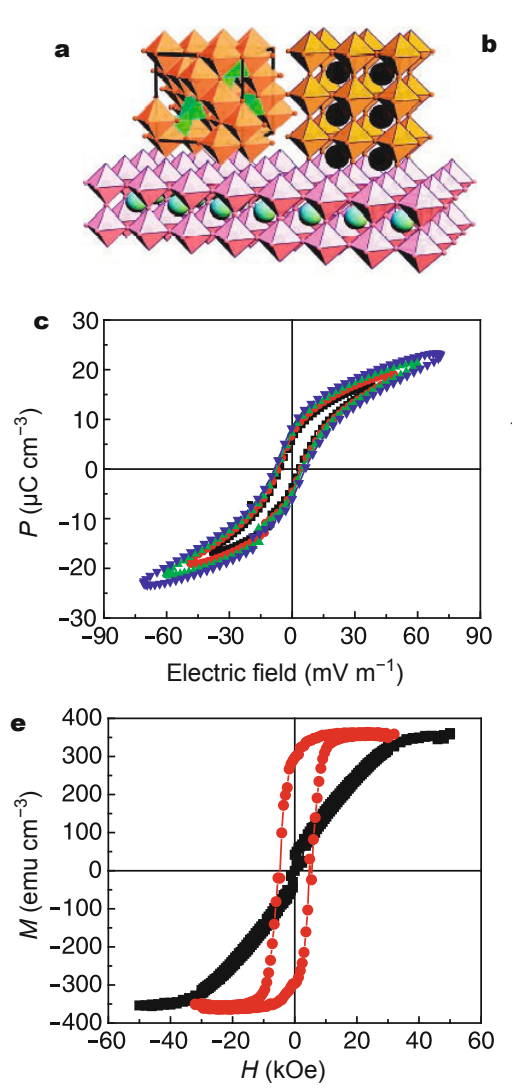
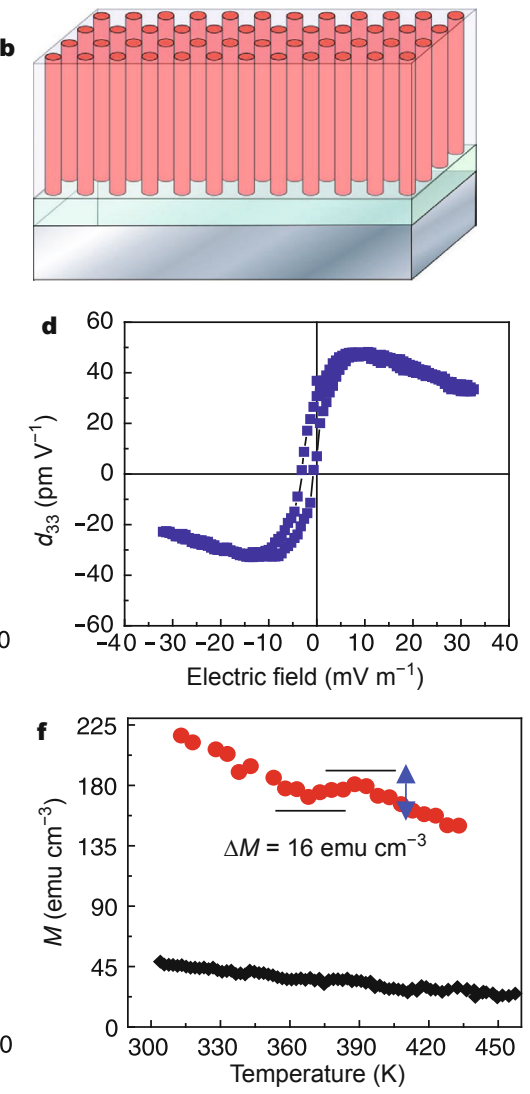

Figure 1 (a) Epitaxial relationship between CFO (top left) and BTO (top right) on a (001)-oriented STO substrate (bottom). (b) Schematic illustration of a self-assembled nanostructure thin film grown on the STO substrate. (c) Ferroelectric hysteresis loops. (d) The typical converse piezoelectric $d_{33}$ hysteresis loop for the nanocomposite. (e) Out-of-plane (red) and in-plane (black) magnetic hysteresis loops of the nanocomposite. (f) Temperature-dependent magnetization measured under a magnetic field of 100 Oe (red curve), showing an obvious coupling between ferroelectric and magnetic orderings (the multilayered nanostructure (black curve) shows negligible change in magnetization). Reprinted with permission from Ref. [19]. Copyright 2004, American Association for the Advancement of Science. age current in the nanostructures inhibits their possible applications. People start to search for more reliable and robust 2-2 horizontal heterostructures. In 2006, epitaxial piezoelectric $\mathrm{PZT}$ was grown on the magnetostrictive single crystal $\mathrm{La}_{1.2} \mathrm{Sr}_{1.8} \mathrm{Mn}_{2} \mathrm{O}_{7}$ by Wu et al. [25]. The strong coupling at interface helped to achieve a considerable $\mathrm{ME}$ response. External magnetic field of $1 \mathrm{~T}$ was applied at the point of ferromagnetic phase transition $(120 \mathrm{~K})$ and a maximum voltage output of $\Delta V_{\mathrm{ME}} \approx 15 \mu \mathrm{V}$ was obtained. The $\mathrm{ME}$ coefficient can be as large as $600 \mathrm{mV} \mathrm{cm}^{-1} \mathrm{Oe}$. In addition, by tuning the frequency of applied alternating current magnetic field in those materials with high magnetostrictive and piezoelectric coefficients, they proposed that it was possible to further improve the magnitude of ME response. In order to achieve a large inverse ME coupling in the 2-2 heterostructures, Eerenstein et al. [26] grew epitaxial $\mathrm{La}_{0.3} \mathrm{Sr}_{0.7} \mathrm{MnO}_{3}$ (LSMO) on BTO single crystals. A large inverse ME coefficient $\left(\sim 2.3 \times 10^{-7} \mathrm{~s} \mathrm{~m}^{-1}\right)$ was observed, which was attributed to the local stress-controlled magnetic anisotropy. The magnetization in LSMO thin film can be controlled by the non- $180^{\circ}$ polarization switching of the BTO single crystal. In rhombohedral $\mathrm{Pb}\left(\mathrm{Mg}_{1 / 3} \mathrm{Nb}_{2 / 3}\right)_{0.72}$ $\mathrm{Ti}_{0.28} \mathrm{O}_{3}$ (PMN-PT) single crystals with high piezoelectricity, Thiele et al. [27] discovered that the $T_{c}$ of LSMO thin film increased by $19 \mathrm{~K}$ and the ME coefficient was about $6 \times 10^{-8} \mathrm{~s} \mathrm{~m}^{-1}$, which further confirmed the effective contribution from the strain transfer across the interface on the ME coupling. In principle, mechanical-stress-controlled $\mathrm{Mn}-\mathrm{O}$ bond length can be assumed as the microscopic origin of the ferromagnetic states of the LSMO thin film.

Liu et al. [28] deposited the $\mathrm{Fe}_{3} \mathrm{O}_{4}$ on a variety of piezoelectric substrates such as $\mathrm{PZT}, \mathrm{Pb}\left(\mathrm{Zn}_{1 / 3} \mathrm{Nb}_{2 / 3}\right) \mathrm{O}_{3-x} \mathrm{PbTiO}_{3}$ (PZN-PT) and PMN-PT by spin-spray deposition. The microwave ME coefficient of the composite was measured as $108 \mathrm{Oe} \mathrm{cm} \mathrm{kV}^{-1}$ on the films grown on PZN-PT. The tunable ferromagnetic resonance field of $\mathrm{Fe}_{3} \mathrm{O}_{4}$ can be enhanced to 860 Oe under the application of the electric field. The magnitude of ferromagnetic resonance field could be dependent on epitaxial mismatch strain and the directions of external magnetic fields. These studies broke new ground in prototype microwave ferromagnetic devices controlled through electric fields.

In 2011, Lahtinen et al. [29] found that the magnetic domain patterns of CoFe could correspond to the ferroelectric domains when the alloy was grown on ferroelectric single crystals. In CoFe/BTO heterostructures, strain coupling across the interface from ferroelastic interaction could stabilize the magnetic uniaxial anisotropy. More- 
over, the experimental results showed that it was possible to electrically switch magnetic domain during the in-plane rotation of the ferroelectric polarization. Nan et al. [30] deposited permalloy ultrathin films on the (011)-oriented PMN-PT substrates and found that the effective magnetization was enhanced about 375 Oe through a high voltage due to the strain and surface-charge-mediated ME coupling. With the increase of the electric field, the magnetic anisotropy became stronger. Similar effect can be observed in Py/YMO herterostructure as well [31]. In 2013, Buzzi et al. [32] observed a $90^{\circ}$ rotation of magnetization due to the switching of out-of-plane ferroelectric polarization in artificial multiferroic nanostructures (arrays of Ni on PMNPT substrates). The magnetization rotation was non-volatile and reversible. The $90^{\circ}$ magnetization rotation has also been reported in $\mathrm{CoFeB} /(011)$-cut PMN-PT by Zhang et al. [33]. In 2014, Ghidini et al. [34] and Yang et al. [35] fabricated the Ni/BTO multilayered capacitors and $\mathrm{Co} /$ PMN-PT heterostructures. They succeeded in achieving a strain-driven non-volatile $180^{\circ}$ rotation of magnetization, which was critical for the application of data storage. Recently, Lei et al. [36] prepared the $\mathrm{CoFeB} / \mathrm{Cu} / \mathrm{Co} / \mathrm{Cu} / \mathrm{IrMn} /$ $\mathrm{Pt}$ nanowires on PZT substrates. They observed that the giant magnetoresistive (GMR) effect and the domain wall (DW) motion were controlled through external electric fields on PZT, as shown in Fig. 2.

In addition to the 1-3 perpendicular and 2-2 horizontal heterostructures, large mechanical-strain-driven ME coupling can also happen in a $0-3$ mosaic nanostructure. The CFO has been embedded into the BTO matrix by a solgel method with a ME response reported by Rafique et al. [37]. The capacitance can be controlled using the magnetic field. What's more, in 0-3 CFO-BFO nanocomposites, the capacitance increased as a function of CFO ratio. Thus the largest ME coefficient was obtained in the sample with a molar ratio of 50-50. Halley et al. [38] found $\mathrm{Cr}_{2} \mathrm{O}_{3}$ epitaxial nanoclusters embedded in a single crystalline $\mathrm{MgO}$ matrix, suffering a compressive strain, showed an original superparamagnetic and superparaelectric phases with an enhanced ME coefficient.

\section{Charge transfer}

In magnetic heterostructures or nanocomposites, the accumulation of interfacial charges (electrons or holes) through the application of an external electric field can play a critical role in the spin structures across the interface. Therefore the electric-field-controlled interfacial charge accumulation could be another effective driving force to achieve the ME coupling. One of the examples of the interfacial-charge-modulated magnetic spin structure was shown in the ultrathin magnetic alloys (FePt or FePd) grown on $\mathrm{MgO}$ substrates [39]. The application of an exter-
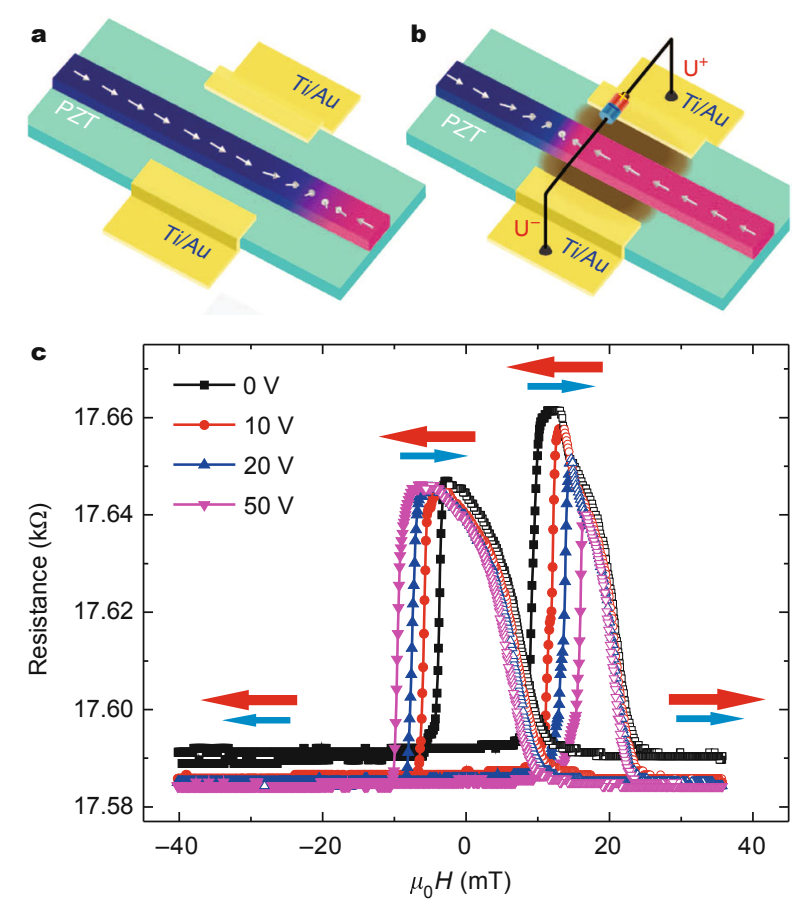

Figure 2 (a) Magnetoresistance as a function of different applied voltages (measurement goes from a depolarized state of the PZT layer). (b) The as-grown DW structures in the magnetic stripe without the application of an external voltage. (c) The strain-mediation DW motion in magnetic stripe modulated by electric fields. Reprinted with permission from Ref. [36]. Copyright 2013, Nature Publication Group.

nal positive/negative bias across the interface of electrolyte (propylene carbonate) and the magnetic ultrathin layers will give rise to the accumulation of holes/electrons. The magnetic coercive field decreases about $4.5 \%$ and increases about $1 \%$ for FePt and FePd layers when a negative bias of $-600 \mathrm{mV}$ is applied, which is attributed to the interfacial control of the unpaired d electrons of alloys near the interface.

This ME coupling due to the interfacial charge effect triggered a series of theoretical work in heterostructures consisting of ferroelectric (dielectric) and ferromagnetic phases. The magnetic spin states can also be determined by the switching of the ferroelectric polarization. Rondinelli et al. [40] predicted the ME effect at the ferroelectric (dielectric)/magnetic interface using the first principles density functional calculation. Across the interface of ferromagnetic $\mathrm{SrRuO}_{3}$ (SRO) and dielectric STO, the ferromagnetism could be controlled by the accumulation or depletion of the spin-up due to the application of the electric field on the dielectric layer. In this theory, a spin capacitance was proposed to provide a brand-new platform for future electronic/spintronic devices. In 2009, Niranjan et al. [41] studied the interfacial ME effect in SRO/BTO heterostructures using the first-principles calculations. The 
spin structure at the interface can be modulated through the switchable ferroelectric polarization of BTO. This ME effect was attributed to the screening of the charges at the interface with opposite ferroelectric polarizations, where the ME coefficient can be obtained as $\alpha_{\mathrm{s}}=2.3 \times 10^{-10} \mathrm{G} \mathrm{cm}^{2}$ $\mathrm{V}^{-1}$. Further theoretical work on the interfacial ME effects has been done in the non-magnetic $\mathrm{KTaO}_{3} / \mathrm{BTO}$ superlattice by Yang et al. [42]. They demonstrated that the accumulation of holes could give rise to an emerging magnetism at the interface due to the polarization-dependent electronic reconstruction near the interface.

Apart from the above theoretical progresses, Molegraaf et al. [43] experimentally observed the strong interfacialcharge-driven ME coupling in the multiferroic LSMO/PZT heterostructures. The ferroelectric polarizations of PZT at the interface can determine the carrier density of LSMO and the transition between its ferromagnetic-metal and antiferromagnetic-insulating states. On top of the metallic LSMO $\left(\sim 10^{21}\right.$ holes $\left.\mathrm{cm}^{-3}\right)$ film with a thickness of $\sim 4 \mathrm{~nm}$, PZT $\left(\mathrm{P}=50 \mu \mathrm{C} \mathrm{cm}^{-2}\right)$ was grown to form a ferroelectric/ manganite interface. The magnetization of LSMO thin film was controlled by the switching of the out-of-plane ferroelectric polarization of PZT, measured by magneto-optic Kerr effect (MOKE) and superconducting quantum interference device (SQUID). When the polarization pointed to the LSMO thin film, it was an interfacial holes depletion state, while the polarization pointed away from the LSMO, it came to a holes accumulation state at the interface with a decrease of magnetization of LSMO. By measuring the ferroelectric polarization and the magnetization at a function of voltage, the ME coefficient was estimated as $0.8 \times 10^{-3} \mathrm{Oe}$ $\mathrm{cm} \mathrm{V}^{-1}$ at $100 \mathrm{~K}$. The interfacial coupling mechanism and spin reconstruction were revealed by using X-ray absorption near edge spectroscopy (XANES) [44], where a quantitative understanding of the relationship between holes depletion/accumulation and $\mathrm{Mn}-\mathrm{O}$ orbital states (high spin $\mathrm{Mn}^{3+}$ and low spin $\mathrm{Mn}^{4+}$ ) were carried out. Dong et al. [45] explored interfacial magnetism and phase transition controlled by the charge accumulation or depletion, showing the reconstruction of the valence states and magnetic moment. Apart from the magnetization, the magnetic coercive field and the exchange coupling can also be controlled by the interfacial ferroelectric polarization in LSMO/PZT heterostructures [46]. In 2013, Cao et al. [47] reported that the magnetism could be determined by the interfacial charge accumulation/depletion states in EuO/BTO/LSMO nanostructures.

In addition, Ju et al. [48] theoretically proposed a tunneling magnetoresistance (TMR) structure consisting of nonmagnetic metal/multiferroic barrier/ferromagnetic metal. By switching the ferroelectric polarization in the tunneling barrier, the magnitude of TMR can be modulated. The spin filter efficiency was strongly affected by exchange splitting of ferromagnets and the asymmetric energy potentials derived from the opposite polarizations at the interface. In 2010, Garcia et al. [49] demonstrated the polarization-dependent TMR up to $-17 \%$ in a $(5 \mathrm{~nm}) \mathrm{Fe} /(1 \mathrm{~nm}) \mathrm{BTO} /$ $(30 \mathrm{~nm})$ LSMO heterostructures at $4.2 \mathrm{~K}$. Typically, spin polarization was the key parameter to control the behavior of the TMR effect [50]. The spin-polarization-dependent tunneling was very sensitive to the interfacial states of Fe/ BTO and BTO/LSMO, which could be controlled by opposite polarizations as shown in Fig. 3. Yin et al. [51] prepared the $\mathrm{LSMO} / \mathrm{BTO} / \mathrm{La}_{0.5} \mathrm{Ca}_{0.5} \mathrm{MnO}_{3}$ (LCMO) tunneling junctions and discovered that the tunneling electroresistance (TER) and TMR effect could be well determined by the interfacial carrier density through the switching of ferroelectric polarization as well.

In 2013, Yi et al. [52] prepared the LCMO/BFO heterostructures by PLD to study the charge-derived magnetism

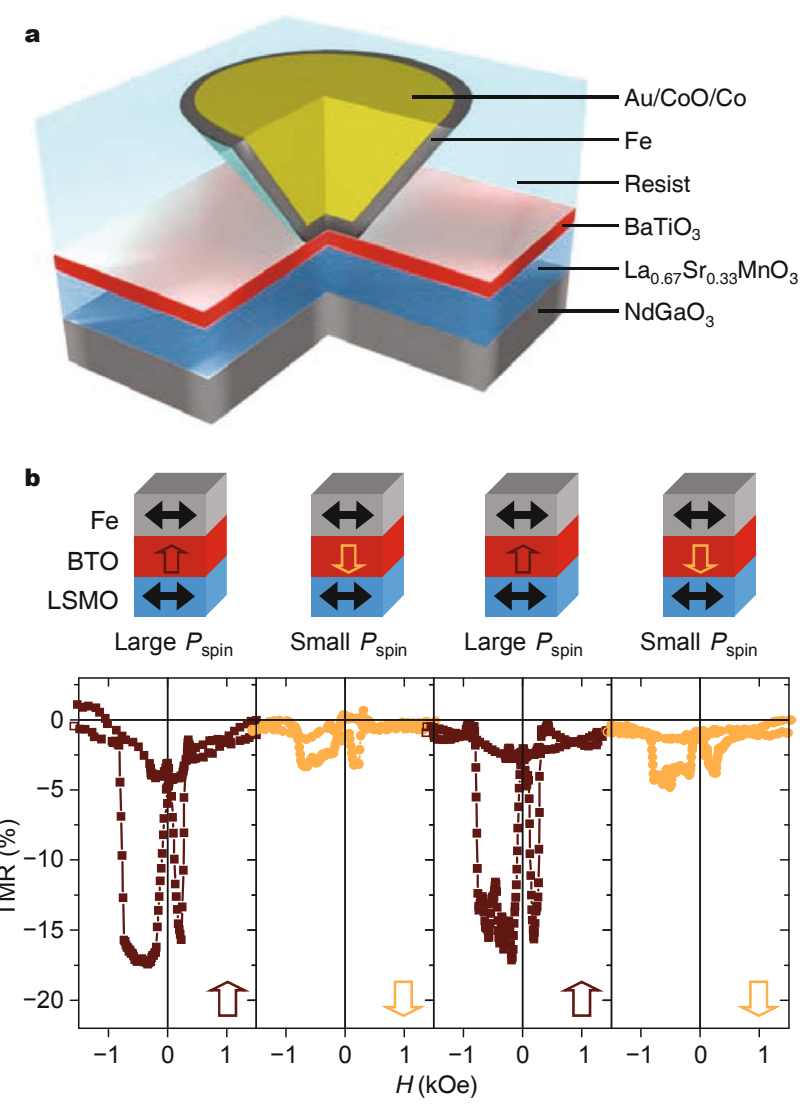

Figure 3 (a) Schematic of the TMR structure. The BTO $(1 \mathrm{~nm}) / \mathrm{LSMO}$ $(30 \mathrm{~nm})$ bilayer is covered with a resist with a nano-hole filled by $\mathrm{Au} /$ $\mathrm{CoO} / \mathrm{Co} / \mathrm{Fe}(5 \mathrm{~nm})$. (b) Under the external voltage of $-50 \mathrm{mV}$, the TMR curves were recorded at the temperature of $4.2 \mathrm{~K}$, when the ferroelectric polarization is up, down, up and down. Reprinted with permission from Ref. [49]. Copyright 2010, American Association for the Advancement of Science. 
of LCMO thin films. When the ferroelectric polarization of $\mathrm{BFO}$ pointed to the LCMO, it resulted in the electron accumulation, while a reversed ferroelectric polarization gave rise to holes accumulation in LCMO. As shown in the $\mathrm{X}$-ray magnetic circular dichroism (XMCD) results in Fig. 4 , the magnetization of LCMO with electrons accumulation is much larger than the one with holes accumulation. The microscopic origin of this interfacial magnetization is revealed by the study of valence states of Mn ions (high spin $\mathrm{Mn}^{3+}$ and low spin $\mathrm{Mn}^{4+}$ ). Furthermore, there is a strong antiferromagnetic coupling between the antiferromagnetic BFO and ferromagnetic LCMO at the interface with holes accumulation, while an enhanced magnetization is observed close to the interface due to the electrons accumulation. In 2014, Kim et al. [53] used scanning transmission electron microscopy (STEM) and electron energy-loss spectroscopy (EELS) to map out the magnetism with an atomic scale across the BFO/LSMO heterostructures before and after the switching of the ferroelectric polarization, further suggesting that the valence states of $\mathrm{Mn}$ ions were controlled by the polarization. Using a scanning

a
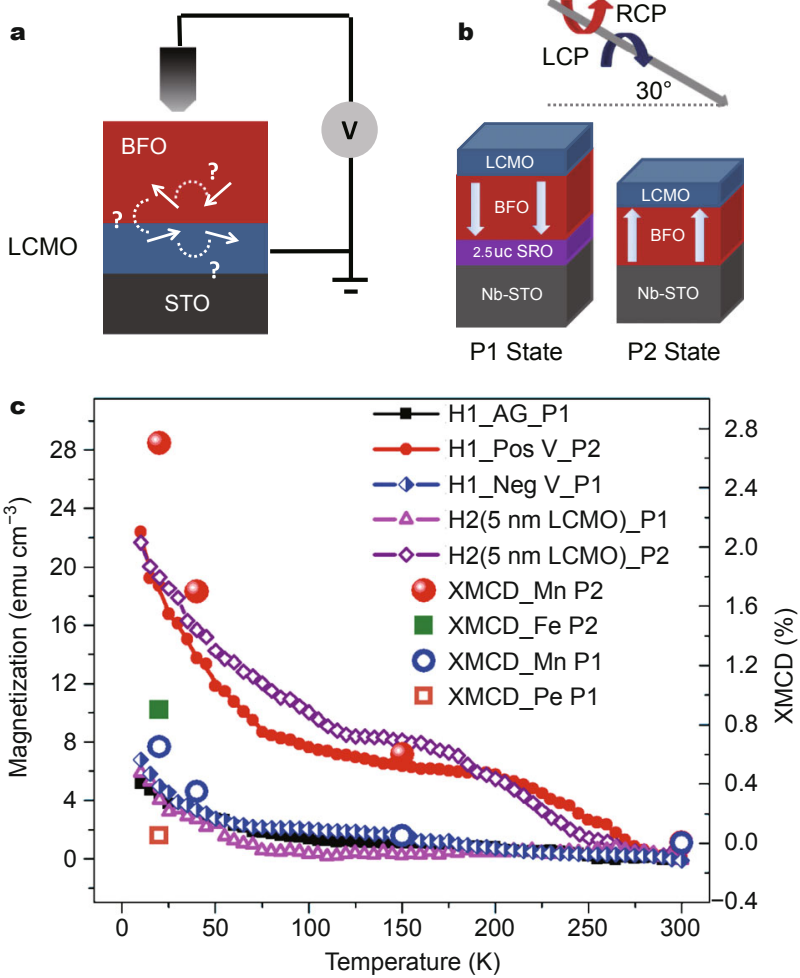

Figure 4 (a) Schematic of the BFO/LCMO (structure H1) heterostructures on STO substrates for ferroelectric switching. (b) Schematic of LCMO/BFO (structure H2) heterostructure used for XAS, XMCD, and XLD measurements. (c) Temperature dependence of magnetization and the spin sum rule of XMCD for Fe atoms and Mn atoms taken at different polarization states. Reprinted with permission from Ref. [52]. Copyright 2013, American Physical Society. probe technique, Wang et al. [54] successfully observed that it was possible to control the spin structures of magnetic DWs in LSMO/dielectric heterostructures. By injecting spin-polarized electrons with an ultralow voltage across the interface of tip and LSMO thin film in the capacitor, the DWs were able to move at the nanoscale. Besides, this modulation could be carried out at ambient conditions. All the above theoretical proposals and experimental discoveries about the emerging magnetism controlled by the interfacial charge may offer a new pathway to achieve the non-volatile spintronic devices at a low energy cost.

\section{Magnetic exchange interaction}

Benefited from the advanced thin film growth techniques, people can build up magnetic systems with exchange interaction across the interface of the high-quality heterostructures. For the $\mathrm{ABO}_{3}$-type perovskite manganites, the double exchange and superexchange interactions between $\mathrm{Mn}$ and $\mathrm{Mn}$ via $\mathrm{O}$ atoms are strongly correlated with the valence states of Mn ions, which further help to determine their ferromagnetic or antiferromagnetic states and their colossal magnetoresistance (CMR) [55]. Recently, people discovered that exotic exchange behaviors occurred in manganite thin films and heterostructures which were absent in their bulk forms [56-59]. The charge transfer and orbital reconstruction can be two of the origins of the degeneration of double exchange or superexchange at the interface. In 2010, Lee et al. [60] utilized the XMCD to study the magnetism of LSMO thin films and found that the remnant magnetization was opposite to the direction of the applied magnetic field. The superexchange coupling between $\mathrm{Mn}$ and $\mathrm{Mn}$ via $\mathrm{O}$ atoms resulted in the electronic reconstruction in the Mn $\mathrm{e}_{\mathrm{g}}$ orbital $\left(\mathrm{d}_{3 z^{2}-r^{2}}\right)$, so that the mixed-valence states of $\mathrm{Mn}^{3+}$ and $\mathrm{Mn}^{4+}$ transformed into $\mathrm{Mn}^{3+}$ enriching state near the LSMO/STO interface. Such a reconstruction gave rise to the antiferromagnetic coupling of $\mathrm{Mn}^{3+}$ perpendicular to the surface direction.

Therefore, via controlling their double exchange or superexchange, it is possible to achieve the ME coupling at the interface of manganite-based heterostructures. In 2006, Chakhalian et al. [61] prepared the superlattice (LCMO and superconducting $\mathrm{YBa}_{2} \mathrm{Cu}_{3} \mathrm{O}_{7}$ (YBCO) alternative layers). They found that the magnetic moments emerged from $\mathrm{Cu}$ atoms were antiparallel to the moment from $\mathrm{Mn}$ atoms at $30 \mathrm{~K}$. This antiferromagnetic coupling was attributed to the superexchange interaction through $\mathrm{Cu} \mathrm{d}_{3 z^{2}-r^{2}}$ electrons hopping into the empty $\mathrm{Mn}^{3+} \mathrm{d}_{3 z^{2}-r^{2}}$ orbital at the LCMO/YBCO interface. They further studied the interfacial electron states in this superlattice and trilayers using X-ray absorption spectroscopy (XAS) and X-ray linear dichroism (XLD) [4], which directly demonstrated the orbital reconstruction of $\mathrm{Cu}$ and $\mathrm{Mn}$ atoms as a result of $\mathrm{Cu}-\mathrm{O}-\mathrm{Mn}$ superexchange 
and thus the interfacial superconducting behavior was suppressed. Garcia-Barriocanal et al. [62] studied the LaM$\mathrm{nO}_{3}$ (LMO)/STO superlattice, where the superexchange in $\mathrm{Ti}-\mathrm{O}-\mathrm{Mn}$ gave rise to the magnetic moment of $\mathrm{Ti}$ atoms and the antiferromagnetic coupling between $\mathrm{Ti}$ and $\mathrm{Mn}$ as well. In 2013, Liu et al. [63] studied the $\mathrm{LCMO} / \mathrm{PrBa}_{2} \mathrm{C}$ $\mathrm{u}_{3} \mathrm{O}_{7}$ (PBCO)/LCMO magnetic tunnel junctions and observed that the magnitude of TMR abruptly decreased at low temperature, which was also related to the thickness of PBCO layer. They regarded this anomalous phenomenon as the spin filter effect at the LCMO/PBCO interface derived from the antiferromagnetic coupling between spin polarization of LCMO and a reversed magnetic moment of PBCO. Based on the studies of Liu et al. [63] and Cuellar et al. [64] explored the detailed mechanism of the interfacial spin interaction in such magnetic tunnel junctions. Figs $5 \mathrm{a}-\mathrm{c}$ show the electric-field-modulated TMR in the LCMO/PBCO/LCMO magnetic tunnel junctions. Super- exchange in $\mathrm{Cu}-\mathrm{O}-\mathrm{Mn}$ at $\mathrm{LCMO} / \mathrm{PBCO}$ interface resulted in the charge transfer and orbital reconstruction with an antiferromagnetic coupling as shown in Fig. 5d. Besides, the strength and decay length of antiferromegnetic coupling in $\mathrm{Cu}$ and $\mathrm{Mn}$ could be modulated using an external voltage and thus the ME effect was achieved.

For these antiferromagnetic materials such as LMO and $\mathrm{SrMnO}_{3}(\mathrm{SMO})$ alternative layers, both experimental results and theoretical calculations demonstrated the emerged interfacial ferromagnetism in their superlattice [65-67]. What's more, Chen et al. [68] theoretically predicted the transition from ferromagnetism to antiferromagnetism by the switching of ferroelectric polarization of $\mathrm{BTO}$ in the SMO/BTO/LMO superlattice due to the double exchange and superexchange at the interfaces.

The magnetic exchange coupling at the interface can also be driven by the orbital reconstruction. In 2010, Yu et al. $[69,70]$ demonstrated that unexpected ferromagnetic
Figure 5 (a) Schematic of the LCMO/PBCO/LCMO magnetic tunnel junction. Magnetic field was applied in-plane along the [110] direction which was the easy axis direction of the top layer. (b) With the application of an external voltage by $100 \mathrm{mV}$ (blue symbols), The two low resistance states represent the saturation state that magnetic moments are aligning to the [110] direction of the top layer and the moments are aligned the easy axis of the bottom layer, respectively. The high resistance state shows the misalignment between top and bottom layer. (c) At the higher bias of $250 \mathrm{mV}$, no significant magnitude of TMR effect is shown in the major loop (black symbols). (d) Model for the antiferromagnetic $\mathrm{Mn}-\mathrm{Cu}$ interaction across the interface. The thick grey line illustrates the $x^{2}-y^{2}$ conduction band profile. Reprinted by permission from Ref. [64]. Copyright 2014, Macmillan Publishers Ltd. a
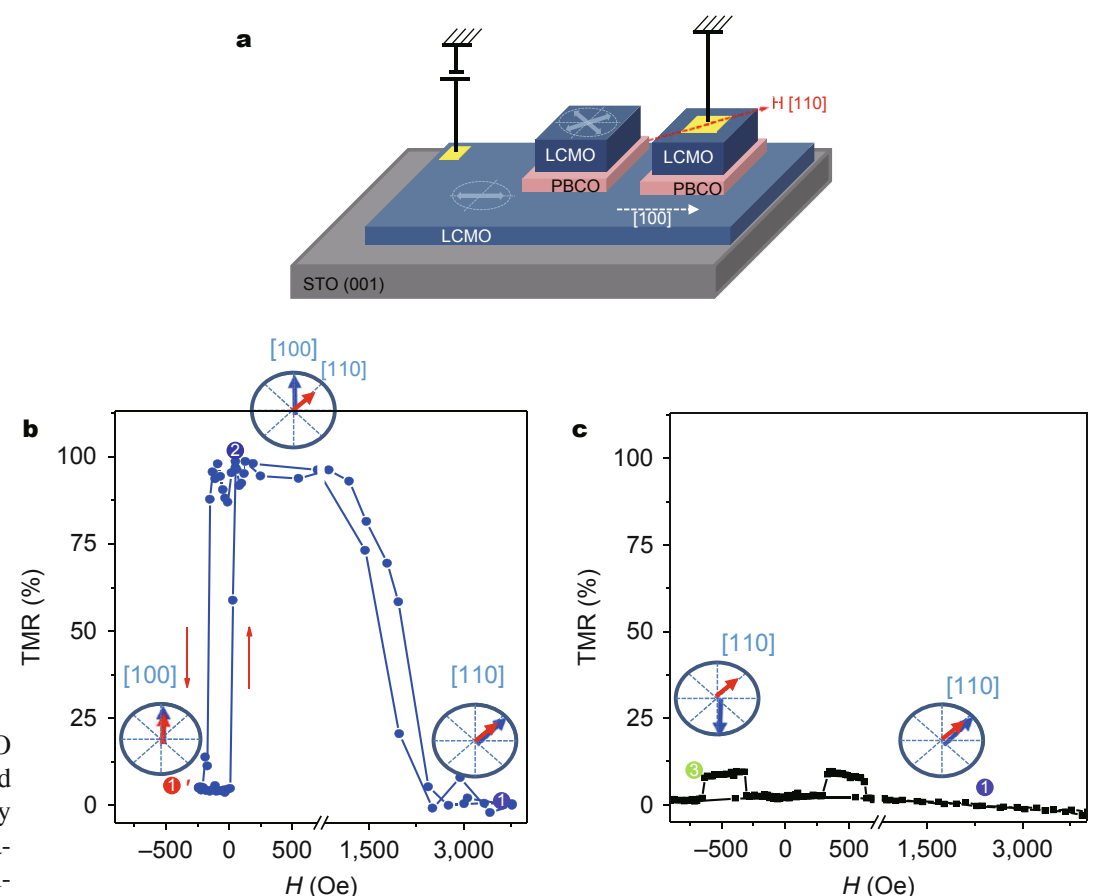

d

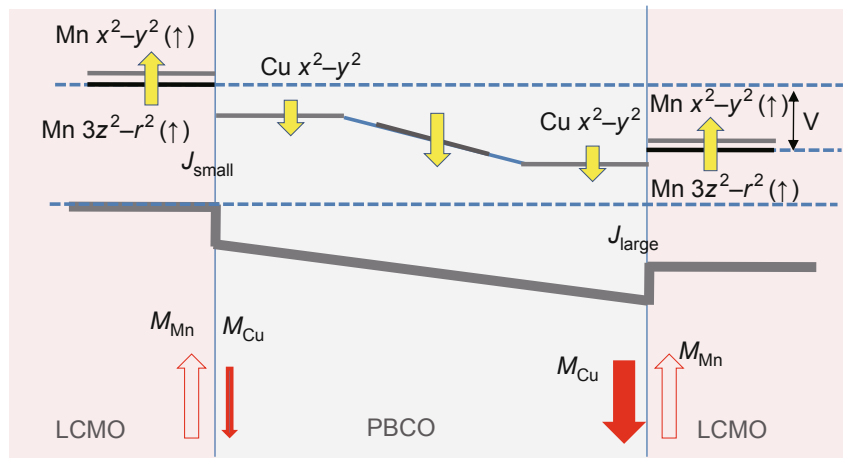


orderings and magnetic exchange bias emerged at the interface of the ferromagnetic LSMO and antiferromagnetic BFO heterostructures, which was confirmed by combined techniques such as SQUID, XMCD and XAS. Orbital reconstruction due to the superexchange between transition metal $\mathrm{Fe}$ ions and multivalent $\mathrm{Mn}$ ions was studied when the perovskite LSMO film was La or Sr terminated. This superexchange through $\mathrm{O}$ atoms resulted in a ferromagnetic order in BFO close to the interface. Thus, the coupling between the induced ferromagnetic BFO and the antiferromagnetic $\mathrm{BFO}$ gave rise to an exchange bias. More importantly, the interfacial exchange bias can be controlled by switching the ferroelectric polarization of the multiferroic BFO. In such a heterostructure, Wu et al. [71,72] demonstrated that the ferroelectric polarization could be coupled with the ferromagnetic order at BFO interface, which was used to control the magnitude of magnetic coercive field and the directions of the exchange bias as shown in Fig. 6. Very recently, Rao et al. [73] succeeded in integrating the $\mathrm{BFO} / \mathrm{LSMO}$ heterostructures on Si substrates with a strong enhancement of magnetization compared with the one of LSMO thin films. The magnitude of the exchange bias was determined by the field cooling. The reversible control of the interfacial multiferroic order parameters due to magnetic exchange interaction offers additional opportunities for the design of future ME electronic devices.

In addition to the double-exchange and superexchange in manganite materials, there exist other magnetic exchange interactions to achieve the ME effect, such as the ferromagnetic and antiferromagnetic exchange coupling near their interfaces. In 2008, Mathur [74] had demonstrated that the magnetic domain structures could be controlled by applying electric field in the multiferroic BFO-based heterostructures. Chu et al. [75] and Heron et al. [76] took CoFe alloy as the ferromagnetic layer to construct a coupling between ferromagnetism and antiferromagnetism in $\mathrm{BFO} /$ CoFe. Using XMCD-photo emission electron microscopy (PEEM) and piezoresponse force microscopy (PFM), Chu found that the magnetic domain structures of CoFe were coupled with the ferroelectric domains and the antiferromagnetic easy plane of BFO. These discoveries showed that the strong magnetic interaction occurred across the ferromagnet-multiferroic interface at room temperature. The magnetization of CoFe layer could be reversibly switched by applying an electric field. Similar effect was also reported in $\mathrm{BFO} / \mathrm{Co}_{40} \mathrm{Fe}_{40} \mathrm{~B}_{20}(\mathrm{CoFeB})$ structures [77].

The ferroelectric polarization is able to be controlled by external magnetic fields as well. In 2012, Rogdakis et al. [78] reported that ferroelectricity emerged in non-ferroelectric $\mathrm{NdMnO}_{3}(\mathrm{NMO}) / \mathrm{SMO} / \mathrm{LMO}$ artificial tri-layer nanostructures. Both LMO and NMO are A-type antiferromagnets with $\mathrm{Mn}^{3+}$ ions, whereas SMO is a G-type antiferromagnet

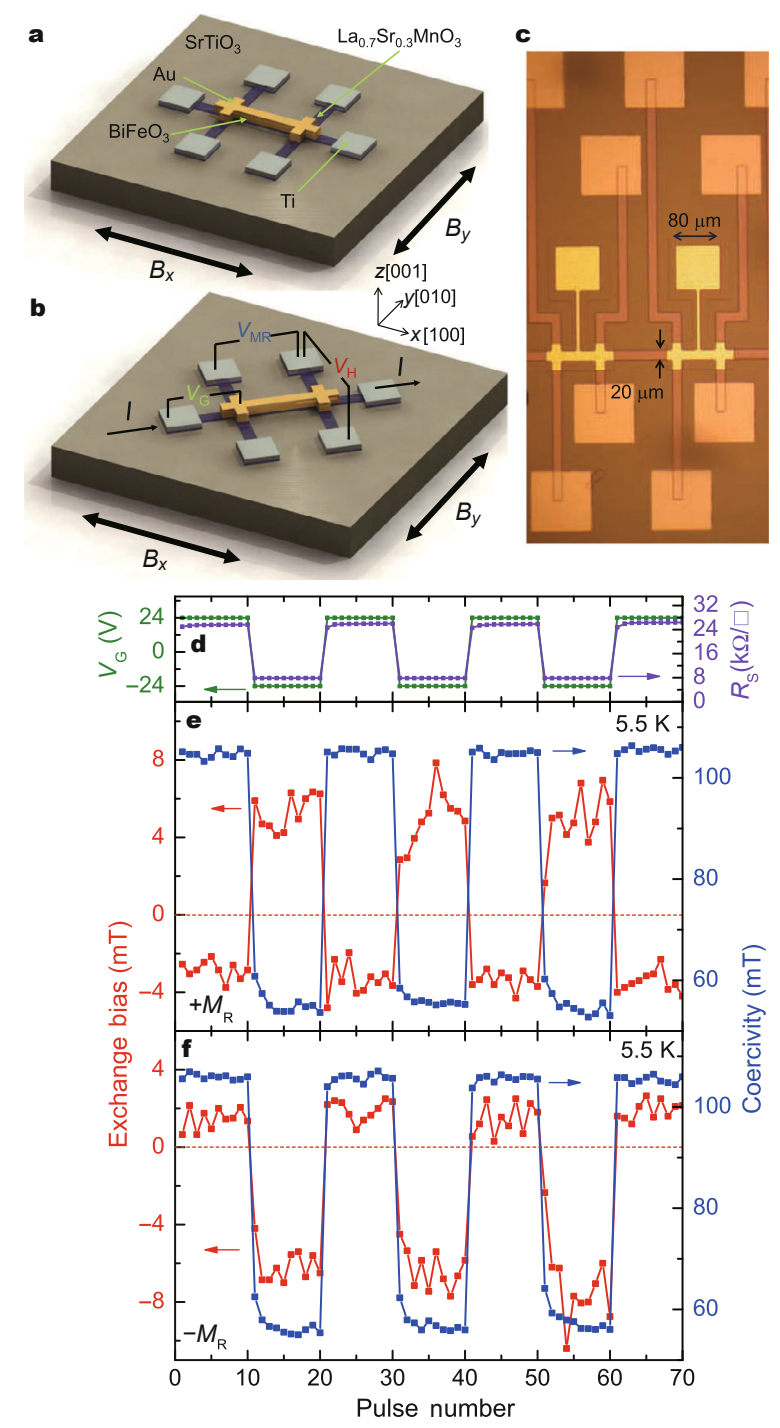

Figure 6 (a, b) Schematics of two devices with structures of gated hall bars. Current is aligned along the [100] direction in (a), while along the [110] direction in (b). Pulsed-voltages are applied to switch the ferroelectric polarization of the BFO and the magnetoresistivity of LSMO is measured under the applied fields. (c) An optical photograph of the device. (d) Pulsed-voltages are applied (green, left arrow) and the sheet resistance of the LSMO (purple, right arrow) correspond to the voltages. (e, f) Exchange bias and coercivity data at the temperature of $5.5 \mathrm{~K}$ with the application of positive and negative pulsed-voltages. Reprinted with permission from Ref. [72]. Copyright 2013, American Physical Society.

with $\mathrm{Mn}^{4+}$ ions [79-81]. According to the first-principles calculations, the exchange coupling between $\mathrm{Mn}^{3+}$ and $\mathrm{Mn}^{4+}$ resulted in the interfacial mixed-valence states and broken space inversion symmetry (interfacial polar discontinuity and cationic asymmetry), which stabilized the electric dipolar moment. Furthermore, external magnetic field triggered a significant enhancement of ferroelectricity about $150 \%$. 


\section{Orbital hydridization}

Except for the ME effect due to the mechanical coupling, interfacial charge transfer and magnetic exchange interaction, the orbital hybridization in the ME nanocomposites was another important parameter which could be used to achieve the ME response. Such an orbital hybridization was proposed at the interface of an ultrathin ferromagnetic metal and a ferroelectric oxide. The spin structure at the interface can be controlled by the ferroelectric polarization. Duan et al. [82] theoretically predicted that the magnetic moment from $\mathrm{Fe}$ atoms and the emerged moment from $\mathrm{Ti}$ atoms were antiparallel with each other in Fe/BTO multilayer structures. The magnetization at the interface was different for the two ferroelectric polarizations (ferroelectric polarization of BTO pointed to the Fe or away from the Fe). The difference of the interfacial magnetization induced by the polarization reversal increased with the thickness of the BTO layer. When Ti atoms displaced to or away from the $\mathrm{O}$ octahedral center in two polarization states, the distances between $\mathrm{Fe}$ and $\mathrm{Ti}$ were different. By orbital-resolved local densities of states (DOS), they predicted the hybridization between Fe and Ti3d orbitals via O2p orbital. The ferroelectric polarization of BTO could be used as a control parameter to tune the interfacial orbital hybridization, which gave rise to the modulation of magnetism at the interface as shown in Fig. 7. In 2011, Yang et al. [83] successfully prepared the Fe/BTO heterostructure on $\mathrm{Pt} / \mathrm{MgO}$ sub- strates using ion beam sputter deposition. Such composite nanostructures presented excellent ferroelectric and ferromagnetic properties at room temperature. The interfacial magnetization in Fe/BTO was larger than the pure Fe thin film due to the orbital hybridization of $\mathrm{Ti}$ and Fe. Later, Radaelli et al. [84] was able to control the ME effect at the interface of BTO and Fe. They prepared the multilayered heterostructures of $\mathrm{Au} / \mathrm{Co} / \mathrm{Fe} / \mathrm{BTO} / \mathrm{LSMO}$ on STO substrates. Through the switching of the ferroelectric polarization, the interfacial magnetic states could be switched. The ME coefficient was about $2 \times 10^{-9} \mathrm{G} \mathrm{cm}^{2} \mathrm{~V}^{-1}$.

In 2012, using the first-principles calculations, Dai et al. [85] proposed that the interfacial magnetization can be switched through the ferroelectric polarization in Ni/BTO superlattice. The interfacial ME coupling mechanisms are different between Fe/BTO and Ni/BTO systems. The interfacial magnetic moments on $\mathrm{Ni}$ atoms were responsible for the coupling in Ni/BTO, whereas in Fe/BTO, the change of induced magnetic moment of interfacial Ti atoms was crucial to the observed $\mathrm{ME}$ effect. Other $3 \mathrm{~d}$ transition systems, such as $\mathrm{Co} / \mathrm{BTO}, \mathrm{Fe}_{3} \mathrm{O}_{4} / \mathrm{BTO}$ and $\mathrm{Co}_{2} \mathrm{MnSi} / \mathrm{BTO}$ systems, were also studied theoretically [86-89]. In 2014, Chen et al. [90] proposed a model and theoretically studied that the magnetization can be controlled via the switching of the ferroelectric polarization in $\mathrm{Co}_{2} \mathrm{FeSi} / \mathrm{BTO} / \mathrm{Co}_{2} \mathrm{FeSi}$ multiferroic tunnel junctions, which was attributed to the multiple orbital hybridizations of Co-Ti and Fe-Ti. Furthermore,
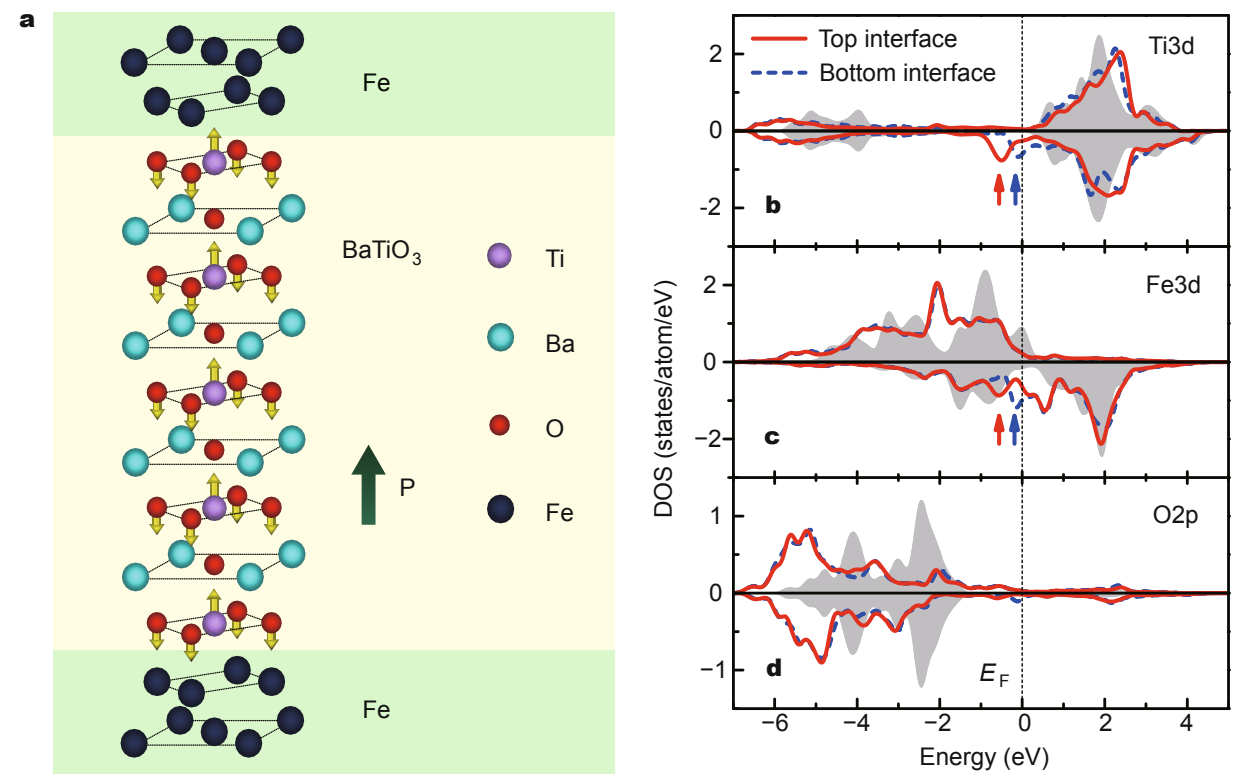

Figure 7 (a) Model of the $\mathrm{Fe} / \mathrm{BaTiO}_{3}$ multilayer for 4 unit cells BTO layer. The ferroelectric displacement of Ti and $\mathrm{O}$ atoms is showed through yellow arrows when the polarization of BTO points up. (b, c, d) The orbital-resolved DOS data for interfacial atoms of Ti3d, Fe3d, and O2p in the Fe/BTO multilayer with 4 unit cells BTO layer. The upper and lower panels represent the spin up and spin down DOS, respectively. The shades show the bulklike DOS of atoms in the central monolayer of (c) Fe or (b), (d) $\mathrm{TiO}_{2}$. The vertical line indicates the Fermi energy $\left(E_{\mathrm{F}}\right)$. Reprinted with permission from Ref. [82]. Copyright 2006, American Physical Society. 

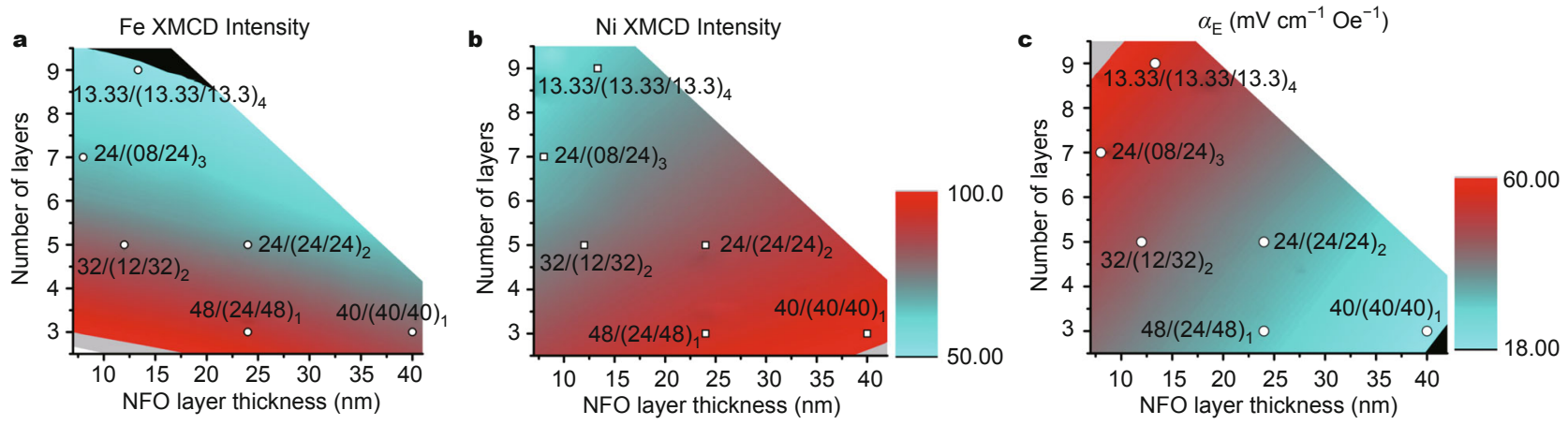

Figure $8(\mathrm{a}, \mathrm{b})$ The layer number $(2 n+1)$ dependent and NFO thickness-dependent XMCD data for $L_{3}$ intensity of Fe and Ni. (c) The ME coupling coefficient $\alpha_{\mathrm{E}}$ under different NFO thickness and layer number. Reprinted with permission from Ref. [91]. Copyright 2014, American Physical Society.

they calculated that the coupling strength was different between $\mathrm{Co}-\mathrm{Ti}$ and $\mathrm{Fe}-\mathrm{Ti}$. The interfacial ME coefficients were about $0.9 \times 10^{-10} \mathrm{G} \mathrm{cm}^{2} \mathrm{~V}^{-1}$ for $\mathrm{Co}_{2} / \mathrm{TiO}_{2}$ and $2.3 \times 10^{-10}$ $\mathrm{G} \mathrm{cm} \mathrm{C}^{2} \mathrm{~V}^{-1}$ for $\mathrm{FeSi} / \mathrm{TiO}_{2}$. Verma et al. [91] prepared the $\mathrm{BTO} /\left(\mathrm{NiFe}_{2} \mathrm{O}_{4} / \mathrm{BTO}\right)_{n}$ multilayers on STO substrates. By controlling the thickness of NFO, the magnetic moment of $\mathrm{Fe}$ atoms remained while the moment of $\mathrm{Ni}$ atoms changed significantly. They confirmed that the mechanism of ME effect in such a multiferroic composite was the hybridization of interfacial Ni3d orbital with BTO electronic states instead of Fe atoms, as shown in Fig. 8.

\section{CONCLUSIONS}

The ME effect remains an important research hotspot in recent decades because it not only has plenty of scientific significance but also has tremendous impacts in areas as diverse as microelectronics, energy-conversion devices and sensors. With the appearance of thin film epitaxial growth techniques (laser molecular beam epitaxy, metal organic chemical vapor deposition, magnetron sputtering, etc.), multiferroic ME nanostructures with exotic physical phenomena due to the coupling of multiple degrees of freedoms, such as lattice, spin, charge and orbital, have drawn more attention for recent years. Taking advantage of multiple advanced characterization tools such as SPM, TEM, XMCD, XAS, XLD, EELS and PEEM, scientists are able to further study and explore the emerging quantum states in those transition metal oxides. Therefore, it is possible to find a way to increase the magnitude of the ME coefficient, and achieve the electric-field modulation of spin structures at room temperature.

Compared with the single-phase multiferroic materials which have been discovered for over a century, multiferroic composites at nanoscale have been studied in depth only for recent decades. Owing to the boarder application prospects, people start to keep an eye on composites and nanostructures. In this review, we introduce the recent progress of the ME coupling in multiferroic nanocompos- ites in terms of the four main mechanisms including strain effect, charge transfer, magnetic exchange interaction and orbital hybridization. Based on these natures of the strong coupling of multiple orderings in those complex oxide materials, scientists may bring into other control factors. For example, additional control parameters such as polarized light or mechanical force can be used to achieve the ME coupling. The application of heat gradient may also be proposed to be another way to develop future electronic nanodevices with multiple functionalities.

Received 26 December 2014; accepted 26 January 2015 published online 10 February 2015

1 Ohtomo A, Hwang HY. A high-mobility electron gas at the $\mathrm{LaAlO}_{3}$ / $\mathrm{SrTiO}_{3}$ heterointerface. Nature, 2004, 427: 423-426

2 Seidel J, Martin LW, He Q, et al. Conduction at domain walls in oxide multiferroics. Nat Mater, 2009, 8: 229-234

3 Lebeugle D, Mougin A, Viret M, Colson D, Ranno L. Electric field switching of the magnetic anisotropy of a ferromagnetic layer exchange coupled to the multiferroic compound $\mathrm{BiFeO}_{3}$. Phys Rev Lett, 2009, 103: 257601

4 Chakhalian J, Freeland JW, Habermeier HU, et al. Orbital reconstruction and covalent bonding at an oxide interface. Science, 2007, 318: 1114-1117

5 Zhang JX, He Q, Trassin M, et al. Microscopic origin of the giant ferroelectric polarization in tetragonal-like $\mathrm{BiFeO}_{3}$. Phys Rev Lett, 2011, 107: 147602

6 Curie P. Sur la symétrie dans les phénomènes physiques, symétrie d'un champ électrique et d'un champ magnétique. J Phys, 1894, 3: 393

7 Debye P. Bemerkung zu einigen neuen Versuchen über einen magneto-elektrischen Richteffekt. Z Phys, 1926, 36: 300-301

8 Landau L, Lifshitz E. Electrodynamics of Continuous Media. Oxford: Pergamon press, 1960

9 Dzyaloshinskii IE. On the magneto-electrical effects in antiferromagnets. Zh Exp Teor Fiz, 1960, 37: 881-882

10 Astrov DN. The magnetoelectric effect in antiferromagnetics. Sov Phys JETP 1960, 11: 708-709

11 Zhao T, Scholl A, Zavaliche F, et al. Electrical control of antiferromagnetic domains inmultiferroic $\mathrm{BiFeO}_{3}$ films at room temperature. Nat Mater, 2006, 5: 823-829

12 Hill NA, Rabe KM. First-principles investigation of ferromagnetism and ferroelectricity in bismuth manganite. Phys Rev B, 1999, 59: 8759 
13 Kimura T, Goto T, Shintani H, et al. Magnetic control of ferroelectric polarization. Nature, 2003, 426: 55-58

14 Fiebig M, Lottermoser T, Fröhlich D, Goltsev AV, Pisarev RV. Observation of coupled magnetic and electric domains. Nature, 2002, 419: $818-820$

15 Rivera JP. On definitions, units, measurements, tensor forms of the linear magnetoelectric effect and on a new dynamic method applied to $\mathrm{Cr}-\mathrm{Cl}$ boracites. Ferroelectrics, 1994, 161: 165-180

16 Prashanthi K, Shaibani PM, Sohrabi A, Natarajan TS, Thundat T. Nanoscale magnetoelectric coupling in multiferroic $\mathrm{BiFeO}_{3}$ nanowires. Phys Status Solidi RRL, 2012, 6: 244-246

17 Kimura T, Kawamoto S, Yamada I, et al. Magnetocapacitance effect in multiferroic $\mathrm{BiMnO}_{3}$. Phys Rev B, 2003, 67: 18041(R)

18 Nan CW. Magnetoelectric effect in composites of piezoelectric and piezomagnetic phases. Phys Rev B, 1994, 50: 6082

19 Zheng $\mathrm{H}$, Wang J, Lofland SE, et al. Multiferroic $\mathrm{BaTiO}_{3}-\mathrm{CoFe}_{2} \mathrm{O}_{4}$ nanostructures. Science, 2004, 303: 661-663

20 von Hippel A, Breckenridge RG, Chesley FG, Tisza L. High dielectric constant ceramics. Ind Eng Chem, 1946, 38: 1097-1109

21 Fritsch D, Ederer C. Epitaxial strain effects in the spinel ferrites $\mathrm{CoFe}_{2} \mathrm{O}_{4}$ and $\mathrm{NiFe}_{2} \mathrm{O}_{4}$ from first principles. Phys Rev B, 2010, 82: 104117

22 Zavaliche F, Zheng H, Mohaddes-Ardabili L, et al. Electric field-induced magnetization switching in epitaxial columnar nanostructures. Nano Lett, 2005, 5: 1793-1796

23 Zheng H, Zhan Q, Zavaliche F, et al. Controlling self-assembled perovskite-spinel nanostructures. Nano Lett, 2006, 6: 1401-1407

24 Zhang JX, Fu H, Lu W, Dai J, Chan HLW. Nanoscale free-standing magnetoelectric heteropillars. Nanoscale, 2013, 5: 6747-6753

25 Wu T, Zurbuchen MA, Saha S, et al. Observation of magnetoelectric effect in epitaxial ferroelectric film/manganite crystal heterostructures. Phys Rev B, 2006, 73: 134416

26 Eerenstein W, Wiora M, Prieto JL, Scott JF, Mathur ND. Giant sharp and persistent converse magnetoelectric effects inmultiferroic epitaxial heterostructures. Nat Mater, 2007, 6: 348-351

27 Thiele C, Dörr K, Bilani O, Rödel J, Schultz L. Influence of strain on the magnetization and magnetoelectric effect in $\mathrm{La}_{0.7} \mathrm{~A}_{0.3} \mathrm{MnO}_{3} /$ PMN-PT(001) (A = Sr, Ca). Phys Rev B, 2007, 75: 054408

28 Liu M, Obi O, Lou J, et al. Giant electric field tuning of magnetic properties in multiferroic ferrite/ferroelectric heterostructures. Adv Funct Mater, 2009, 19: 1826-1831

29 Lahtinen THE, Tuomi JO, van Dijken S. Pattern transfer and electric-field-induced magnetic domain formation in multiferroic heterostructures. Adv Mater, 2011, 23: 3187-3191

30 Nan TX, Zhou ZY, Liu M, et al. Quantification of strain and charge co-mediated magnetoelectric coupling on ultra-thin permalloy/ PMN-PT interface. Sci Rep, 2014, 4: 3688

31 Laukhin V, Skumryev V, Marti X, et al. Electric-field control of exchange bias in multiferroic epitaxial heterostructures. Phys Rev Lett, 2006, 97: 227201

32 Buzzi M, Chopdekar RV, Hockel JL, et al. Single domain spin manipulation by electric fields in strain coupled artificial multiferroic nanostructures. Phys Rev Lett, 2013, 111: 027204

33 Zhang S, Zhao Y, Xiao X, et al. Giant electrical modulation of magnetization in $\mathrm{Co}_{40} \mathrm{Fe}_{40} \mathrm{~B}_{20} / \mathrm{Pb}\left(\mathrm{Mg}_{1 / 3} \mathrm{Nb}_{2 / 3}\right)_{0.7} \mathrm{Ti}_{0.3} \mathrm{O}_{3}(011)$ heterostructure. Sci Rep, 2014, 4: 3727

34 Ghidini M, Pellicelli R, Prieto JL, et al. Non-volatile electrically driven repeatable magnetization reversal with no applied magnetic field. Nat Commun, 2013, 4: 1453

35 Yang SW, Peng RC, Jiang T, et al. Non-volatile $180^{\circ}$ magnetization reversal by an electric field in multiferroic heterostructures. Adv Mater, 2014, 26: 7091-7095

36 Lei N, Devolder T, Agnus G, et al. Strain-controlled magnetic domain wall propagation in hybrid piezoelectric/ferromagnetic structures. Nat Commun, 2013, 4:1378
37 Rafique M, ul Hassan SQ, Awan MS, Manzoor S. Dependence of magnetoelectric properties on the magnetostrictive content in 0-3 composites. Ceram Int, 2013, 39: 213-216

38 Halley D, Najjari N, Majjad H, et al. Size-induced enhanced magnetoelectric effect and multiferroicity in chromium oxide nanoclusters. Nat Commun, 2014, 5: 3167

39 Weisheit M, Fähler S, Marty A, et al. Electric field-induced modification of magnetism in thin-film ferromagnets. Science, 2007, 315: 349-351

40 Rondinelli JM, Stengel M, Spaldin NA. Carrier-mediated magnetoelectricity in complex oxide heterostructures. Nat Nanotechnol, 2008, 3: 46-50

41 Niranjan MK, Burton JD, Velev JP, Jaswal SS, Tsymbal EY. Magnetoelectric effect at the $\mathrm{SrRuO}_{3} / \mathrm{BaTiO}_{3}(001)$ interface: an ab initio study. Appl Phys Lett, 2009, 95: 052501

42 Yang Y, Lin CS, Chen JF, Hu L, Cheng WD. Magnetoelectric effects at the interfaces between nonmagnetic perovskites: $a b$ initio prediction. EPL, 2014, 105: 27002

43 Molegraaf HJA, Hoffman J, Vaz CAF, et al. Magnetoelectric effects in complex oxides with competing ground states. Adv Mater, 2009, 21: $3470-3474$

44 Vaz CAF, Hoffman J, Segal Y, et al. Origin of the magnetoelectric coupling effect in $\mathrm{Pb}\left(\mathrm{Zr}_{0.2} \mathrm{Ti}_{0.8}\right) \mathrm{O}_{3} / \mathrm{La}_{0.8} \mathrm{Sr}_{0.2} \mathrm{MnO}_{3}$ multiferroic heterostructures. Phys Rev Lett, 2010, 104: 127202

45 Dong S, Zhang X, Yu R, Liu JM, Dagotto E. Microscopic model for the ferroelectric field effect in oxide heterostructures. Phys Rev B, 2011, 84: 155117

46 Preziosi D, Fina I, Pippel E, et al. Tailoring the interfacial magnetic anisotropy in multiferroic field-effect devices. Phys Rev B, 2014, 90: 125155

47 Cao S, Liu P, Tang J, et al. Magnetoelectric coupling at the EuO/ $\mathrm{BaTiO}_{3}$ interface. Appl Phys Lett, 2013, 102: 172402

48 Ju S, Cai TY, Guo GY, Li ZY. Electrically controllable spin filtering and switching in multiferroic tunneling junctions. Phys Rev B, 2007, 75: 064419

49 Garcia V, Bibes M, Bocher L, et al. Ferroelectric control of spin polarization. Science, 2010, 327: 1106-1110

50 Jullière M. Tunneling between ferromagnetic films. Phys Lett A, 1975, 54: 225-226

51 Yin YW, Burton JD, Kim YM, et al. Enhanced tunnelling electroresistance effect due to a ferroelectrically induced phase transition at a magnetic complex oxide interface. Nat Mater, 2013, 12: 397-402

52 Yi D, Liu J, Okamoto S, et al. Tuning the competition between ferromagnetism and antiferromagnetism in a half-doped manganite through magnetoelectric coupling. Phys Rev Lett, 2013, 111: 127601

53 Kim YM, Morozovska A, Eliseev E, et al. Direct observation of ferroelectric field effect and vacancy-controlled screening at the Bi$\mathrm{FeO}_{3} / \mathrm{La}_{x} \mathrm{Sr}_{1-x} \mathrm{MnO}_{3}$ interface. Nat Mater, 2014, 13: 1019-1025

54 Wang J, Xie LS, Wang CS, et al. Magnetic domain-wall motion twisted by nanoscale probe-induced spin transfer. Phys Rev B, 2014, 90: 224407

55 Khomskii DI. Orbital effects in manganites. Int J Mod Phys B, 2001, 15: 2665-2681

56 Cui B, Song C, Li F, et al. Tuning the entanglement between orbital reconstruction and charge transfer at a film surface. Sci Rep, 2014, 4: 4206

57 Cossu F, Schwingenschlögl U, Colizzi G, Filippetti A, Fiorentini V. Surface antiferromagnetism and incipient metal-insulator transition in strained manganite films. Phys Rev B, 2013, 87: 214420

58 Tebano A, Aruta C, Sanna S, et al. Evidence of orbital reconstruction at interfaces in ultrathin $\mathrm{La}_{0.67} \mathrm{Sr}_{0.33} \mathrm{MnO}_{3}$ films. Phys Rev Lett, 2008, 100: 137401

59 Dong S, Yunoki S, Zhang X, et al. Highly anisotropic resistivities in the double-exchange model for strained manganites. Phys Rev B, 2010, 82: 035118 
60 Lee JS, Arena DA, Yu P, et al. Hidden magnetic configuration in epitaxial $\mathrm{La}_{1-x} \mathrm{Sr}_{x} \mathrm{MnO}_{3}$ films. Phys Rev Lett, 2010, 105: 257204

61 Chakhalian J, Freeland JW, Srajer G, et al. Magnetismat the interface between ferromagnetic and superconducting oxides. Nat Phys, 2006, 2: 244-248

62 Garcia-Barriocanal J, Cezar JC, Bruno FY, et al. Spin and orbital T magnetism at $\mathrm{LaMnO}_{3} / \mathrm{SrTiO}_{3}$ interfaces. Nat Commun, 2010, 1: 82

63 Liu YH, Cuellar FA, Sefrioui Z, et al. Emergent spin filter at the interface between ferromagnetic and insulating layered oxides. Phys Rev Lett, 2013, 111: 247203

64 Cuellar FA, Liu YH, Salafranca J, et al. Reversible electric-field control of magnetization at oxide interfaces. Nat Commun, 2014, 5: 4215

65 Adamo C, Ke X, Schiffer P, et al. Electrical and magnetic properties of $\left(\mathrm{SrMnO}_{3}\right)_{n} /\left(\mathrm{LaMnO}_{3}\right)_{2 n}$ superlattices. Appl Phys Lett, 2008, 92 : 112508

66 Bhattacharya A, May SJ, Te Velthuis SGE, et al. Metal-insulator transition and its relation to magnetic structure in $\left(\mathrm{LaMnO}_{3}\right)_{2 n}$ l $\left(\mathrm{SrMnO}_{3}\right)_{n}$ superlattices. Phys Rev Lett, 2008, 100: 257203

67 Nanda BRK, Satpathy S. Electronic and magnetic structure of the $\left(\mathrm{LaMnO}_{3}\right)_{2 n} /\left(\mathrm{SrMnO}_{3}\right)_{n}$ superlattices. Phys Rev B, 2009, 79: 054428

68 Chen LY, Chen CL, Jin KX, Wu T. Prediction of giant magnetoelectric effect in $\mathrm{LaMnO}_{3} / \mathrm{BaTiO}_{3} / \mathrm{SrMnO}_{3}$ superlattice: the role of n-type $\mathrm{SrMnO}_{3} / \mathrm{LaMnO}_{3}$ interface. J Appl Phys, 2014, 116: 074102

69 Yu P, Lee JS, Okamoto $S$, et al. Interface ferromagnetism and orbital reconstruction in $\mathrm{BiFeO}_{3}-\mathrm{La}_{0.7} \mathrm{Sr}_{0.3} \mathrm{MnO}_{3}$ heterostructures. Phys Rev Lett, 2010, 105: 027201

70 Yu P, Chu YH, Ramesh R. Emergent phenomena at multiferroic heterointerfaces. Phil Trans R Soc A, 2012, 370: 4856-4871

71 Wu SM, Cybart SA, Yu P, et al. Reversible electric control of exchange bias in a multiferroic field-effect device. Nat Mater, 2010, 9: 756-761

72 Wu SM, Cybart SA, Yi D, et al. Full electric control of exchange bias. Phys Rev Lett, 2013, 110: 067202

73 Rao SS, Prater JT, Wu F, et al. Interface magnetism in epitaxial Bi$\mathrm{FeO}_{3}-\mathrm{La}_{0.7} \mathrm{Sr}_{0.3} \mathrm{MnO}_{3}$ heterostructures integrated on $\mathrm{Si}$ (100). Nano Lett, 2013, 13: 5814-5821

74 Mathur N. A desirable wind up. Nature, 2008, 454: 591-592

75 Chu YH, Martin LW, Holcomb MB, et al. Electric-field control of local ferromagnetism using a magnetoelectric multiferroic. Nat Mater, 2008, 7: 478-482

76 Heron JT, Trassin M, Ashraf K, et al. Electric-field-induced magnetization reversal in a ferromagnet-multiferroic heterostructure. Phys Rev Lett, 2011, 107: 217202

77 Béa $\mathrm{H}$, Bibes $\mathrm{M}$, Ott $\mathrm{F}$, et al. Mechanisms of exchange bias with multiferroic $\mathrm{BiFeO}_{3}$ epitaxial thin films. Phys Rev Lett, 2008, 100: 017204

78 Rogdakis K, Seo JW, Viskadourakis Z, et al. Tunable ferroelectricity in artificial tri-layer superlattices comprised of non-ferroic compo- nents. Nat Commun, 2012, 3: 1064

79 Töpfer J, Goodenough JB. $\mathrm{LaMnO}_{3+\delta}$ revisited. J Solid State Chem, 1997, 130: 117-128

80 Chatterji T, Ouladdiaf B, Bhattacharya D. Neutron diffraction investigation of the magnetic structure and magnetoelastic effects in $\mathrm{NdMnO}_{3}$. J Phys Condens Matter, 2009, 21: 306001

81 Wollan E, Koehler W. Neutron diffraction study of the magnetic properties of the series of perovskite-type compounds $[(1-x) \mathrm{La}$, $x$ Ca] $\mathrm{MnO}_{3}$. Phys Rev, 1955, 100: 545

82 Duan CG, Jaswal SS, Tsymbal EY. Predicted magnetoelectric effect in $\mathrm{Fe} / \mathrm{BaTiO}_{3}$ multilayers: ferroelectric control of magnetism. Phys Rev Lett, 2006, 97: 047201

83 Yang P, Zhu JS, Lee JY, Lee HY. Thin film processing and multiferroic properties of $\mathrm{Fe}-\mathrm{BaTiO}_{3}$ hybrid composite. Trans Nonferrous Met Soc China, 2011, 21: 92-95

84 Radaelli G, Petti D, Plekhanov E, et al. Electric control of magnetism at the $\mathrm{Fe} / \mathrm{BaTiO}_{3}$ interface. Nat Commun, 2014, 5: 3404

85 Dai JQ, Zhang H, Song YM. Interfacial electronic structure and magnetoelectric effect in $\mathrm{M} / \mathrm{BaTiO}_{3}(\mathrm{M}=\mathrm{Ni}, \mathrm{Fe})$ superlattices. J Magn Magn Mater, 2012, 324: 3937-3943

86 Sante DD, Yamauchiandand K, Picozzi S. Beyond standard local density approximation in the study of magnetoelectric effects in $\mathrm{Fe} /$ $\mathrm{BaTiO}_{3}$ and $\mathrm{Co} / \mathrm{BaTiO}_{3}$ interfaces. J Phys Condens Matter, 2013, 25: 066001

87 Niranjan MK, Velev JP, Duan CG, Jaswal SS, Tsymbal EY. Magnetoelectric effect at the $\mathrm{Fe}_{3} \mathrm{O}_{4} / \mathrm{BaTiO}_{3}(001)$ interface: a first-principles study. Phys Rev B, 2008, 78: 104405

88 Yamauchi K, Sanyaland B, Picozzi S. Interface effects at a half-metal/ ferroelectric junction. Appl Phys Lett, 2007, 91: 062506

89 Chen LY, Chen CL, Jin KX, Du XJ. Potential enhancement in magnetoelectric effect at $\mathrm{Mn}$-rich $\mathrm{Co}_{2} \mathrm{MnSi} / \mathrm{BaTiO}_{3}$ (001) interface. EPL, 2012, 99: 57008

90 Chen J, Lin C, Yang Y, Hu L, Cheng W. Ab initio study of the magnetoelectric effect and critical thickness for ferroelectricity in $\mathrm{Co}_{2} \mathrm{FeSi} /$ $\mathrm{BaTiO}_{3}$ multiferroic tunnel junctions. Modelling Simul Mater Sci Eng, 2014, 22: 015008

91 Verma VK, Singh VR, Ishigami K, et al. Origin of enhanced magnetoelectric coupling in $\mathrm{NiFe}_{2} \mathrm{O}_{4} / \mathrm{BaTiO}_{3}$ multilayers studied by X-ray magnetic circular dichroism. Phys Rev B, 2014, 89: 115128

Acknowledgements This work was supported by the National Natural Science Foundation of China (51322207 and 51332001), Beijing Natural Science Foundation (2132023) and the Fundamental Research Funds for the Central Universities (2012LYB07).

Author contributions Yao X and Ma J co-wrote the paper. Zhang J, Lin $\mathrm{Y}$ and $\mathrm{Nan} \mathrm{C}$ were involved in the discussion.

Conflict of interest The authors declare that they have no conflict of interest. 


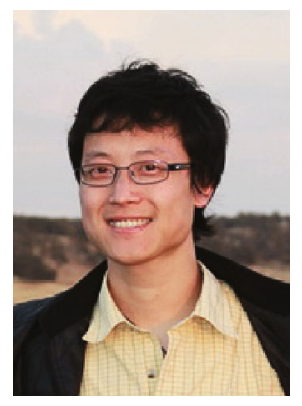

Jinxing Zhang obtained his PhD from Hong Kong Polytechnic University in 2009 under the supervision of Prof. Helen Chan. After that he continued his research work at the Department of Physics of University of California, Berkeley as a post-doc scholar at Professor R. Ramesh's group. In 2012, he joined the Department of Physics, Beijing Normal University as a professor. The central goal of Zhang's group is the pursuit of the emerging phenomena and exotic physical behaviors behind the coupling and control of multiple order parameters (e.g., lattice, spin, orbital, charges) at a reduced dimension. His team is striving to create a bridge between those fundamentally scientific discoveries in functional nano-systems and future possible applications such as sensing, actuation, data storage, energy conversion, etc.

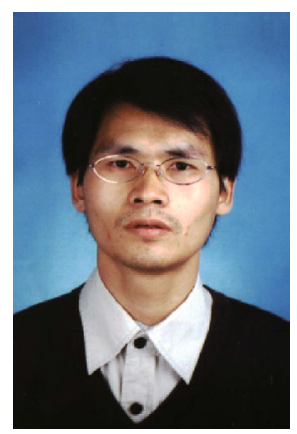

Yuanhua Lin is "Changjiang Scholar" distinguished professor of Materials Science at the School of Materials Science and Engineering, Tsinghua University. He received his BSc degree from the East China Institute of Technology, and his MSc degree from the Institute of Process and Engineering, Chinese Academy of Sciences, and his PhD degree from Tsinghua University. He was a Japan Society for the Promotion of Science scholar at the University of Tokyo in 2005. His main research interests include: high $\mathrm{k}$ ceramics and thin films for high energy density capacitors applications; oxides-based DMS thin films; high-temperature oxides thermoelectric materials and devices for energy conversion.

中文摘要 近几十年来, 人们对于下一代多功能磁电器件(例如换能器, 传感器等)的需求越来越高, 因此吸引了众多科学家探索与研 究多铁性材料的磁电耦合效应, 在室温以及低维条件下寻求电与磁的强耦合. 由于单相多铁性材料的磁电耦合强度非常弱, 或者耦合 温度远远低于室温, 科学家们开始设计和研究多铁性复合材料及其纳米结构. 本综述将从应力、电荷转移、磁交换作用, 以及轨道杂 化四个方面介绍多铁性复合材料磁电效应的耦合机制. 通过充分了解这些序参量在磁电耦合效应中的作用, 我们能够成功实现纳米尺 度下外电场(磁场)对磁性能(铁电性能)的调控. 此外, 这些人造多功能纳米复合材料也为设计自组装纳米结构以及将来实现多功能器 件的应用提供了广阔的平台. 